\title{
WRS/WARISATA

\section{Conocimiento acerca de las didácticas de enseñanza del inglés como lengua extranjera de los docentes de un programa bilingüe de primaria}

\author{
Knowledge regarding didactics on english teaching as a foreign language by \\ elementary teachers
}

Palabras clave: Inglés como lengua extranjera; bilingüismo; didáctica del inglés

\section{RESUMEN}

La formación del docente en el siglo XXI, en especial del que enseña inglés como lengua extranjera, es un reto producto de la globalización y de los desafíos y desarrollo que se experimenta en el mundo actual. Esta investigación tuvo como objetivo caracterizar el conocimiento acerca de las didácticas de enseñanza del inglés como lengua extranjera, que poseen los docentes de un programa bilingüe de primaria de un colegio bilingüe del este de Caracas. Se trató de una investigación descriptiva, con un diseño transeccional, contemporáneo, de campo. Los resultados obtenidos indicaron que la formación en didáctica de los docentes de inglés que trabajan en educación básica en el colegio objeto de estudio es regular, pero casi la mitad de los docentes tiene una formación entre muy deficiente. El mayor énfasis formativo está en las orientaciones y en las actividades de supervisión que reciben de la institución.

\footnotetext{
ABSTRACT

Teacher training in the 21st century, especially those who teach English as a foreign language, is a challenge that is the product of globalization and the challenges and development that is experienced in today's world. The objective of this research was to characterize the knowledge about the didactics of teaching English as a foreign language, which the teachers of a bilingual elementary program of a bilingual school in eastern Caracas possess. It was a descriptive investigation, with a transectional, contemporary, field design. The results obtained indicated that the didactic training of English teachers working in basic education at the school under study is regular, but almost half of the teachers have very poor training. The greatest training emphasis is on the guidance and supervisory activities they receive from the institution.
} 


\section{INTRODUCCIÓN}

El aprendizaje de un segundo idioma o de un idioma extranjero podría resultar un reto para las personas que quisieran alcanzar el mismo nivel de su lengua materna en el otro idioma de su elección. Ciertamente, el aprendizaje de una nueva lengua se facilita cuando la persona se encuentra en el país en el que se habla la lengua meta ya que se ve en la obligación de desenvolverse en la cultura de este nuevo idioma. Sin embargo, cuando se está en un ambiente donde la lengua que se habla es distinta a la meta, así como en el contexto del aula, el proceso de aprendizaje se torna más difícil y complejo por lo que requiere de docentes capacitados que no solo dominen la lengua $y$ todos los componentes, sino que posea las herramientas necesarias para hacer del proceso enseñanza-aprendizaje una experiencia exitosa.

Por lo general, los estudiantes se sienten motivados al entrar en contacto con otra lengua, pero esta motivación puede desaparecer si deben hacer un esfuerzo exagerado para lograr el aprendizaje esperado y si no cuentan con una didáctica apropiada.

Para Hernán y Garcés (2006), los currículos contemporáneos plantean la necesidad de enseñar inglés en edades cada vez más tempranas. La adquisición de este idioma como lengua extranjera, requiere por parte de los maestros un reciclaje continuo y un cambio consciente en la forma de dar la clase. La incorporación de un planteamiento relacionado con la forma como se organiza la información, el material y los recursos que se usan, así como la conciencia de la forma en que aprenden los niños es tan importante como las técnicas que se utilizan.

Los niños aportan a la clase ciertas destrezas, habilidades y sobre todo una imaginación desbordante que, puede ayudarles a adquirir en los primeros ciclos de primaria un idioma nuevo. Los responsables educativos, los padres y los maestros son conscientes de ello y como resultado, la edad en que los niños empiezan a aprender inglés es cada vez más temprana, mientras que el nivel que deben exigir los docentes ha ido aumentando.

Enseñar inglés en edades tempranas conlleva a una gran responsabilidad (Hearn y Garcés, 2006). Los maestros deben tener presente que los niños crecen y recuerdan a los profesores que tuvieron influencia en ellos ya sea negativa o positivamente. La enseñanza a niños requiere dedicación y entusiasmo además de un alto nivel de energía para poder estimular y motivarles constantemente. Sobre todo, la enseñanza requiere una conciencia de que todos los niños son diferentes y tienen diferentes formas de aprender, pero que ninguna de ellas es mejor que otra, y son los docentes los que están allí para enseñarles a todos una educación integral. El reconocimiento de esto requiere que el docente se capacite para encontrar nuevas técnicas y métodos de enseñanza.

Por ello, la presente investigación tuvo como propósito identificar las fortalezas y debilidades de los docentes en cuanto a su conocimiento de la didáctica del inglés, para fomentar en los docentes una actitud crítica y reflexiva de su práctica pedagógica, sobre todo con respecto al uso de métodos, estrategias, enfoques, entre otros, de enseñanza de lenguas extranjeras.

En el marco de la problematización de la temática estudiada, fue oportuno considerar que los nuevos contextos culturales surgidos a partir de la globalización, exigen el aprendizaje de varios idiomas, y particularmente del inglés como lengua extranjera y por ello las 
instituciones donde se imparten dichos programas, recurren a procesos de enseñanza dirigidos al dominio de esta lengua. Algunos de ellos se ajustan a los programas desarrollados en países en los cuales el inglés es el idioma oficial, pero otros han desarrollado sus propios programas. Evidencia de la importancia del inglés como lengua extranjera es el hecho de que en el ámbito económico y en la Comunidad Europea el inglés se ha convertido en la principal lengua de comunicación para los ciudadanos. Sin embargo, todavía existen importantes diferencias en el conocimiento y la utilización del inglés entre los países del Norte y el Sur de Europa (Martínez, 2003).

Según Díaz (2009), Latinoamérica también considera el aprendizaje del inglés una necesidad. Igualmente se es consciente de que el dominio del inglés ayuda al manejo de otras áreas del currículo, satisface la exigencia de las universidades para poder realizar estudios superiores, así como las exigencias del mercado laboral, en lo tecnológico, lo científico y lo cultural. Por último, favorece la formación de estructuras sólidas de pensamiento y de competencias ciudadanas y científicas, y estimula el espíritu investigativo. Más aún, tal como lo plantea Díaz (2009), el nuevo conocimiento es producido y publicado en revistas de investigación, en la Internet y en libros mayormente en ese idioma, y a veces pasan varios años antes de contar con ese nuevo conocimiento en español. En un contexto en donde el saber crece a pasos agigantados, uno o dos años resulta mucho tiempo para el acceso a esa nueva información y a sus aplicaciones en realidades como la nuestra que necesitan avanzar quemando etapas.

El manejo de un segundo idioma abre los horizontes intelectuales, para aceptar nuevas culturas y nuevas formas de vida. La adquisición del inglés es imprescindible porque establece puentes de comunicación entre las personas que hablan diferentes idiomas. Además, éste es considerado a nivel mundial, como el idioma que sirve como punto de enlace entre las diferentes naciones (Bracho y Castro, 2007).

Dada la importancia del dominio de un segundo idioma, varios países de América Latina han iniciado agresivas acciones de preparación de profesores y de impulso del aprendizaje de una de estas lenguas entre los niños y jóvenes de la educación. En el caso de Chile, en el 2004, el gobierno emitió una ordenanza que expresa que el currículum debe incluir estrategias que incorporen la formación en competencias claves para mejorar la vida social cultural y laboral de los jóvenes; entre ellas, el aprendizaje de un idioma extranjero. Así mismo, Costa Rica es considerada un país pionero en la región ya que estableció el inglés como materia básica de primaria y secundaria en 1994. Ahora bien, en este país todavía se enfrentan significativos desafíos de calidad y pertinencia. Por ejemplo, dos terceras partes de los docentes de inglés apenas dominan el idioma, mientras que sólo un $11,4 \%$ de los estudiantes que finalizan la secundaria logran un nivel de dominio adecuado (Banco Interamericano de Desarrollo, 2011).

A pesar de la importancia del dominio del idioma inglés, y del esfuerzo de los diversos países, existen todavía muchas dificultades. En el caso de Perú, el porcentaje de la población que habla un segundo idioma internacional es muy reducido, ya que en el diseño curricular oficial el inglés sólo aparece con dos horas a la semana en la educación secundaria, lo cual es insuficiente para siquiera obtener una base elemental de dominio del idioma, y porque son escasos los programas de formación de profesores en lenguas extranjeras (Díaz, 2009). 
En los centros educativos de Venezuela también se han detectado deficiencias en el proceso enseñanzaaprendizaje del inglés. Los maestros que trabajan con niños, adolescentes y adultos encuentran que a menudo sus estudiantes no poseen las destrezas básicas para comprender libros de texto del nivel del grado que cursan, dedican poco tiempo a la lectura por placer y tienen dificultad para escribir resúmenes y participar en intercambios verbales complejos (Bracho y Castro, 2007).

Esta responsabilidad recae en los docentes que laboran en colegios bilingües. Son ellos quienes deben estar al tanto de los procesos de cómo esos alumnos adquieren el lenguaje del segundo idioma para así poder emplear estrategias idóneas para que esa adquisición se logre de manera apropiada atendiendo a la edad, el grado y el nivel de inglés que ya poseen. El docente debe conocer el proceso psicológico del lenguaje del niño, para luego vincular el mismo con el aprendizaje de un segundo idioma y a su vez, con el desarrollo del lenguaje como tal.

Uno de los factores clave para satisfacer la necesidad de una enseñanza de calidad del inglés, lo constituye la formación del personal docente que asume esta responsabilidad. En consecuencia, los sistemas educativos de los diferentes países del mundo dentro de sus políticas educativas han estado promocionando la formación de profesionales de las lenguas extranjeras (Medina, 2009).

En algunos los países latinoamericanos existen problemas con la capacitación de los docentes. Por ejemplo en Perú, el Ministerio de Educación estuvo evaluando la posibilidad de incrementar el número de horas de inglés en la estructura curricular de dos a cuatro horas a la semana y se enfrentó a tres problemas: un currículo sobrecargado de contenidos, la falta de profesores para cubrir ese incremento de carga horaria, y el problema de calidad de formación de profesores. Incluso los centros educativos privados tienen muchas dificultades para conseguir profesores que tengan un manejo elevado del inglés.

Otros países en América Latina están trabajando para la capacitación de sus profesores de inglés. Al respecto México sigue una estrategia de envío de profesores que imparten inglés para ser capacitados en universidades norteamericanas (Díaz, 2009). Otro de los países que están en la vanguardia de desarrollar proyectos buscando una mejora en la calidad de la enseñanza del idioma inglés es Colombia, el cual estableció en el año 2007 programa llamado Colombia bilingüe. El Programa Nacional de Bilingüismo busca que en seis años los docentes alcancen un nivel alto en el dominio del inglés (Díaz, 2009).

Venezuela no escapa a esta realidad. En investigaciones realizadas en la Universidad del Zulia, Salazar y Batista (2005), expone que las prácticas en la enseñanza de las lenguas extranjeras, en general, han sido el producto de los aciertos y desaciertos de paradigmas pasados, que con el pasar de los años, se ha ido relegando a un segundo plano. En Venezuela particularmente, se ha adoptado una constante aceptación de nuevos métodos en la enseñanza del inglés como lengua extranjera, con la intención de mejorar la calidad en los procesos de enseñanza, pero sin conocer la efectividad de los mismos.

De los planteamientos anteriores se deduce que países como Venezuela, no pueden perder tiempo. Se requiere cuanto antes iniciar el diseño de una estrategia de democratización progresiva del dominio del inglés entre los estudiantes de la educación básica. Dicha estrategia supondrá determinar en qué grados educativos focalizar el programa, cómo y de dónde 
saldrán los profesores que se encargarán del dictado de las clases, así como determinar la modalidad de su oferta, si dentro de la estructura curricular básica o como un programa complementario, fuera de la jornada escolar, al cual se accede voluntariamente. Pero sobre todo requerirá de docentes capacitados para la enseñanza integral del inglés.

Los requerimientos de personal capacitado son mayores si la unidad educativa es bilingüe, ya que ésta requiere de un experto mucho más calificado, que maneje además del inglés, los contenidos en dicho idioma. Según Bracho y Castro (2007), es en el ámbito de la educación privada que el inglés toma mayor importancia, ya que se observa que un gran número de estos colegios son los que imparten inglés con muchas horas de dedicación al área. De este grupo, los colegios que más horas de inglés ofrecen son los colegios bilingües inglés-español, los cuales tienen como objetivo formar personas competentes en ambos idiomas y en consecuencia, imparten clases con el mismo contenido en ambos idiomas y con el mismo número de horas.

En Venezuela hay colegios que imparten la formación académica desde muy temprana edad, por tanto tienen sus propios programas curriculares, entre ellos se encuentran los colegios bilingües en inglés en la ciudad de Caracas. Estos centros educativos poseen programas $y$ métodos de enseñanza de la lectura y la escritura en inglés con adaptaciones realizadas de textos foráneos de países donde el inglés es la lengua materna, específicamente con un enfoque utilizado en los Estados Unidos. Estos métodos han tenido que sufrir cambios y modificaciones por parte de los docentes, para lograr la competencia en el idioma inglés dentro del contexto en que se están utilizando.
En la Unidad Educativa que formó parte de este estudio, la instrucción es bilingüe. Los niños comienzan a tener contacto con el inglés desde los 3 años de edad. Entran a Nursery, luego a los 4 años pasan a prekinder, a los 5 años entran a kinder y a los 6 años pasan a cursar lo que para el colegio es first grade (primer grado). De allí pasan a un primer grado que para el colegio es llamado second grade (segundo grado) comenzando con ello la educación básica o primaria, donde se inicia propiamente el desarrollo de ambos idiomas, el materno siendo en este caso el español y el extranjero el inglés. Dicha etapa termina con el sexto grado que dentro del programa bilingüe sería seventh grade (séptimo grado).

No cabe duda de que, en la educación bilingüe, la actualización permanente de los docentes representa una alternativa para ayudar a estos profesionales, en formación y en servicio, a superar sus debilidades, tanto en su competencia lingüística como metodológica, en el proceso de enseñanza de lenguas.

A pesar de la importancia del inglés, todavía el aprendizaje del mismo en la escuela pública comienza sólo en la escuela secundaria. Sin embargo, actualmente hay una tendencia en la escuela pública de bajar la edad en la cual el niño debe aprender la lengua extranjera.

En el caso particular de Venezuela, Bracho y Castro (2007) plantean que en el Programa de Estudio y Manual del Docente de la Tercera Etapa de Educación Básica en la asignatura Inglés, vigente desde 1987, se establecen dos necesidades que orientan el estudio de este idioma: "(1) ofrecer al alumno otra lengua que le permita comunicarse con individuos de otros pueblos y (2) proporcionar al alumno un instrumento para el acceso directo a fuentes de conocimiento científico, tecnológico y humanístico" (Ministerio de 
Educación, 1987 como se citó en Bracho y Castro, 2007).

Para Martínez (2010), el bilingüismo en los primeros años de vida es muy complejo, y para ello deben tomarse en cuenta muchas consideraciones, debido a que el propio proceso del lenguaje materno está en plena formación de manera rápida. Además implica que la persona tenga la capacidad de comprender el sentido de lo que los demás pretenden comunicar por medios verbales o escritos.

En este sentido, Smith citado por Martínez (2010) considera que un niño es bilingüe cuando su nivel de familiarización con la nueva lengua le permite hablar, leer, escribir, su lengua y la extranjera con exactitud y facilidad, y adicionalmente aprende a hacer uso de los símbolos y signos verbales y escritos a manera de garantizar un efectivo proceso de comunicación.

Martínez (2010), enfatiza que para que sea un centro educativo sea bilingüe debe tener peso significativo el número de horas utilizadas para la enseñanza de esa segunda lengua, y debe haber una compensación interna entre una lengua y otra lengua respecto a los contenidos que se imparten en cada una de ellas. Dedicar un número similar de horas en una lengua como en la otra no es suficiente. También es importante que los contenidos sean semejantes.

En consecuencia, debido a la necesidad que tienen las escuelas bilingües de igualar la cantidad de horas y contenidos en inglés y en español, deben incorporar el enfoque de currículo transversal, como punto de partida para integrar la enseñanza de la lengua extranjera con la enseñanza de contenidos, entendiendo como currículo el conjunto de objetivos, contenidos, métodos y criterios de evaluación de cada uno de los niveles, etapas, ciclos, grados y modalidades del sistema educativo que regulan la práctica docente (Hernán y Garcés, 2006).

En el caso de la enseñanza de la lengua extranjera la didáctica tiene un rol fundamental, por lo tanto, para que se pueda lograr un adecuado aprendizaje del inglés como lengua extranjera, es necesario contar con una didáctica integradora, acorde con las necesidades del niño y del contexto en el cual está inmerso.

Medina y Mata (2002), teóricos de la didáctica, la definen como una disciplina pedagógica aplicada, comprometida con la solución de problemas prácticos, que atañen al proceso de enseñanza aprendizaje y a desarrollo profesional de los docentes. Para ellos la didáctica tiene una proyección práctica, ligada a los problemas concretos de docentes y estudiantes, y debe responder a los siguientes interrogantes: para qué formar a los estudiantes, qué mejora profesional necesita el profesorado, quienes son nuestros estudiantes y cómo aprenden, qué se ha de enseñar y que implica la actualización del saber, y especialmente, cómo y con qué medios se puede realizar la tarea de enseñanza.

Para que un docente sea capaz de diseñar una didáctica efectiva es necesario que conozca las tareas que permiten desarrollar las destrezas necesarias para el dominio de la lengua extranjera. A la adquisición de una destreza se le llama enabling skills (Hernán y Garcés, 2006).

La enseñanza del inglés como lengua extranjera pasa necesariamente por encontrar respuestas claras para adecuar del proceso de enseñanza-aprendizaje a las cambiantes realidades que viven el docente y el estudiante (Medina y Mata, 2002). Por esta razón no basta con hacer énfasis en el aprendizaje de los estudiantes, sino también en la formación de los docentes. 
Desde el enfoque del currículo transversal Brewster et al (2002) consideran que el docente de lenguas extranjeras debe estar formado en los siguientes aspectos:

a) Tener la habilidad de analizar y pensar en los tópicos necesarios para planificar las lecciones, planificar el lenguaje que va a ser trabajado y los objetivos del lenguaje, en términos del vocabulario necesario, los patrones gramaticales $y$ las funciones del lenguaje, las cuales necesitan ser modeladas y practicadas.

b) Tener la capacidad de analizar el lenguaje requerido para las actividades y tareas en términos de destrezas o estrategias las cuales necesitan ser modeladas y practicadas.

c) Tener la habilidad para llevar a cabo el conocimiento sobre el lenguaje o el proceso de lenguaje y emplear este conocimiento cuando se realicen actividades y tareas.

d) Comprender los principios y la práctica utilizando una amplia variedad de actividades que apoyen el aprendizaje en los niveles apropiados.

e) La capacidad de establecer actividades de manera que puedan ser tomadas de las destrezas y del desarrollo del lenguaje en las actividades previas para proveer el reciclaje del lenguaje y la continuidad de las tareas.

f) Debe tener conocimiento del manejo del aprendizaje de manera tal que los factores claves tales como la organización y los grupos de monitoreo ayuden a apoyar el aprendizaje independiente donde sea apropiado.

Por otra parte, según Smith citado por Cooper (2006), un maestro bien entrenado debe manejar cuatro áreas para ser eficaz en el logro de los objetivos del aprendizaje: a) Dominio del conocimiento teórico acerca del proceso de aprendizaje.

b) Actitudes favorables hacia el aprendizaje y relaciones humanas genuinas.

c) Dominio de la materia que se va a enseñar.

d) Conocimiento de las técnicas de enseñanza.

Las destrezas anteriores están contenidas en lo que Smith (citado por Cooper 2006) Ilama conocimiento de las técnicas de enseñanza.

Ahora bien, la formación en didáctica abarca el conjunto de actividades que el docente ha desarrollado con el fin de prepararse lo mejor posible para aplicar la didáctica en el aula durante la enseñanza del inglés. Esto incluye el entrenamiento formal $e$ informal que el docente ha realizado, la motivación que recibe por parte de sus formadores y de la institución, las orientaciones recibidas. y el estudio y la lectura como parte del aprendizaje autogógico, la vinculación a redes que le proporcionen acompañamiento y asesoría, la supervisión que recibe de sus empleadores $y$ de sus pares, y las actividades que le permiten actualizarse y alcanzar mayor dominio de la didáctica.

\section{METODOLOGÍA}

Se asumió el tipo de investigación descriptiva, la cual es concebida por Hurtado (2010) como aquella que se ocupa de identificar las características y manifestaciones de un evento de estudio. En cuanto al diseño, de acuerdo a la fuente, es de campo, porque se recogió información directamente del grupo de profesores del programa de inglés. Según la temporalidad, el diseño es contemporáneo porque los datos se recolectaron en el momento en el que el investigador fue testigo de las situaciones, y 
también fue transeccional, tal como lo definen Hernández, Fernández y Batista (2010), porque se estudió el evento en un único momento del tiempo. Por último, de acuerdo a la amplitud de la información, fue univariable.

La población estuvo conformada por todas las docentes de inglés que trabajan en educación básica en el colegio objeto de estudio, ubicado en Valle Arriba, Caracas, en total 9 docentes de género femenino entre 24 y 48 años de edad, para quienes el inglés es una lengua extranjera (no es su lengua materna). Se escogió ésta unidad educativa porque se trata de una institución bilingüe, con 36 años de experiencia en el país. Debido a que la población era pequeña, no se necesitó de la realización de un muestreo.

Para obtener información sobre la formación de los docentes acerca de la didáctica de la enseñanza del inglés se utilizó la técnica de encuesta y se aplicó un cuestionario de formación en didáctica. Este constaba de 34 preguntas que indagaban sobre varios aspectos de la formación Para procesar los datos se utilizaron técnicas de estadística descriptiva como la mediana, las frecuencias y los porcentajes. Se utilizó la mediana debido a que el grupo era pequeño y los resultados no se ajustaban a la distribución de la curva normal.

\section{RESULTADOS}

Para el análisis de la formación de los docentes acerca de la didáctica de la enseñanza del inglés se transformaron los puntajes brutos arrojados por el cuestionario, y se obtuvo, para cada docente, un puntaje transformado en una escala de 0 a 50 puntos. Se calcularon la mediana, el mínimo, el máximo y los cuartiles, y se elaboró el gráfico de caja. Para interpretar los puntajes obtenidos se utilizó la Tabla 1.

Tabla 1. Categorías de formación acerca de la didáctica de la enseñanza del inglés

\begin{tabular}{ll}
\hline Intervalo & Categoría \\
\hline $0-9,9$ & Muy deficiente \\
$10-19,9$ & Deficiente \\
$20-29,9$ & Regular \\
$30-39,9$ & Suficiente \\
$40-50$ & Excelente \\
\hline
\end{tabular}

El análisis global de la formación didáctica se basa en los puntajes totales obtenidos por cada docente en el Cuestionario de Formación elaborado para la presente investigación. La mediana obtenida por el grupo de docentes en el evento formación en didáctica fue de 21,88 puntos sobre 50 (la mitad de los docentes obtuvo puntajes por debajo de 21,88 puntos y la otra mitad por encima), y se ubicó en la categoría regular, lo que significa que los docentes investigados tienen cierta formación acerca de didáctica del inglés, pero ésta no llega a ser suficiente (entre 30 y 40 puntos sobre 50 ). 
Tabla 2. Mediana de formación docente en didáctica

\begin{tabular}{lll}
\hline Formación en didáctica & \\
\hline Mediana & & 21,88 \\
Mínimo & 9 \\
Máximo & 34 \\
& 25 & 10,94 \\
Percentiles & 50 & 21,88 \\
& 75 & 25,00 \\
\hline
\end{tabular}

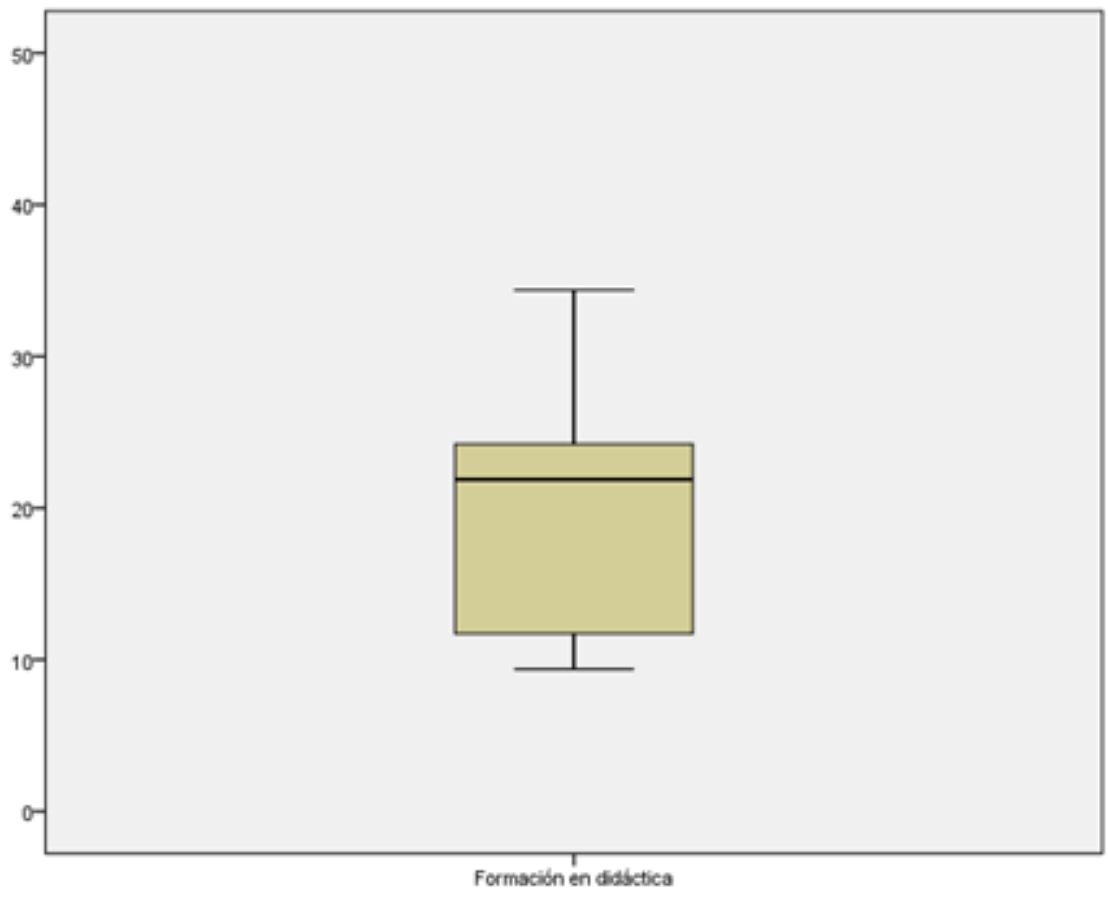

Gráfico 2. Gráfico de caja y bigote de la Formación en didáctica

El $44,4 \%$ de los docentes tienen una formación entre muy deficiente y deficiente, la mayoría $(44,4 \%)$ tiene una formación regular, y apenas un 11,1\% tiene una formación suficiente. Ningún docente obtuvo un puntaje excelente.

Tabla 3. Mediana de formación docente en las dimensiones de formación didáctica

\begin{tabular}{|c|c|c|c|c|c|c|}
\hline & Entrenamiento & Orientaciones & $\begin{array}{c}\text { Modelaje y } \\
\text { monitoreo }\end{array}$ & $\begin{array}{c}\text { Lectura de } \\
\text { material y } \\
\text { textos }\end{array}$ & $\begin{array}{c}\text { Redes y } \\
\text { compartir de } \\
\text { experiencias }\end{array}$ & $\begin{array}{c}\text { Motivación } \\
\text { para } \\
\text { formarse }\end{array}$ \\
\hline Mediana & 11,54 & 37,50 & 41,67 & 16,67 & 8,33 & 33,33 \\
\hline Mínimo & 4 & 6 & 8 & 0 & 0 & 17 \\
\hline Máximo & 31 & 50 & 50 & 50 & 33 & 33 \\
\hline 25 & $5 \quad 5,77$ & 21,88 & 20,83 & 8,33 & 20,83 & 33,33 \\
\hline Percentiles 50 & 11,54 & 37,50 & 41,67 & 16,67 & 41,67 & 33,33 \\
\hline 75 & $5 \quad 16,67$ & 50,00 & 50,00 & 41,67 & 50,00 & 33,33 \\
\hline
\end{tabular}




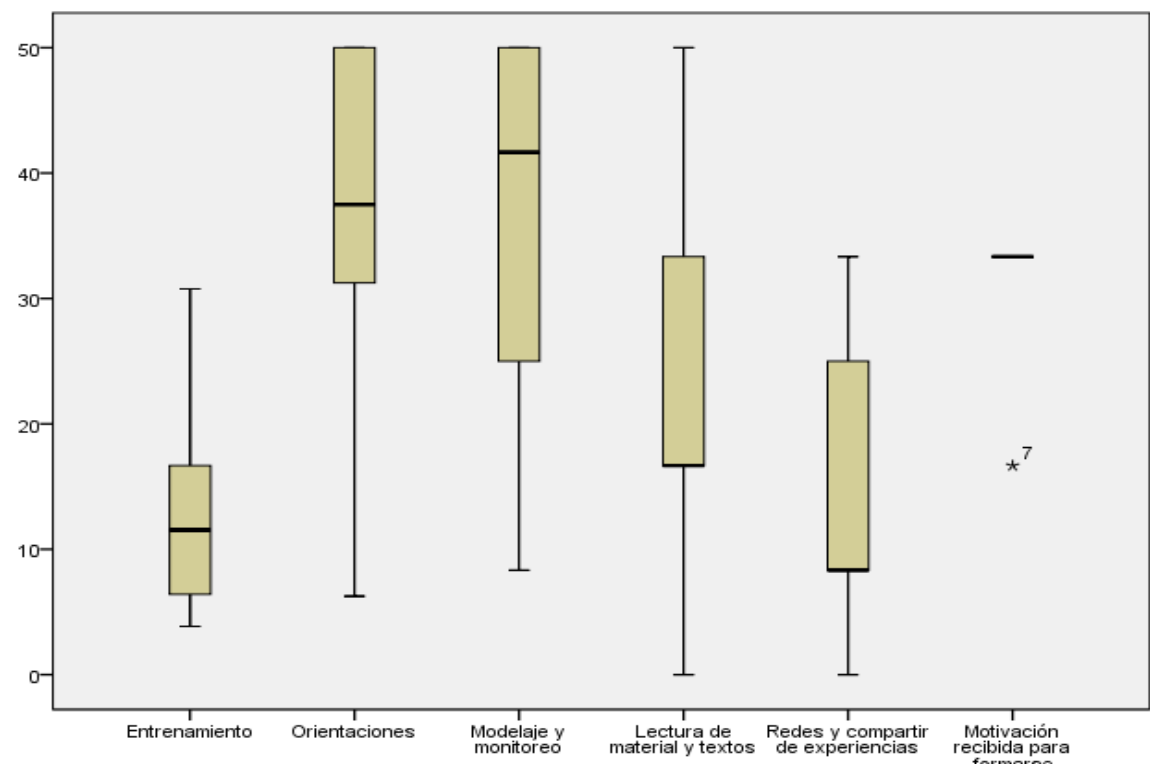

Gráfico 3. Gráfico de caja y bigote de las dimensiones de Formación en didáctica

La Tabla de las medianas, y el gráfico de caja y bigote de las dimensiones de formación muestran que la dimensión con el puntaje más bajo fue Redes y compartir de experiencias, con una mediana de 8,33, la cual se ubicó en la categoría deficiente, seguida por la dimensión entrenamiento (mediana de 11, 54 puntos sobre 50), también ubicada en la categoría deficiente. Las dimensiones donde el grupo de docentes obtuvo las medianas más altas fueron Modelaje y monitoreo $(41,67)$, y Orientaciones $(37,50)$, la primera ubicada en la categoría excelente y la segunda en la categoría suficiente.

La dimensión Entrenamiento se refiere a todas aquellas actividades formativas en las cuales el docente ha participado con la finalidad de recibir conocimientos y destrezas relacionadas con la didáctica del inglés, ya sean académicas formales o informales, tales como cursos, talleres y programas de entrenamiento entre otros. En esta dimensión la mediana se ubicó en 11,54 puntos, en la categoría deficiente, lo cual significa que en general el grupo de docentes ha participado en pocas actividades formativas que le permitan tener dominio de la didáctica para la enseñanza del inglés como lengua extranjera. El puntaje mínimo obtenido fue 4 puntos sobre 50 y lo cual se ubica en la categoría muy deficiente, mientras que el máximo fue 31 y está en el límite inferior de la categoría suficiente. La altura de la caja en el gráfico, muestra que la distribución de la dimensión entrenamiento es relativamente homogénea (los docentes se parecen bastante entre sí en esta característica).

Las actividades de entrenamiento en didáctica general y en el idioma inglés, en las que los docentes más han participado son recibir formación en el idioma inglés (89\%) -aunque sólo el $56 \%$ ha realizado cursos de inglés en instituciones de idiomas en el extranjero-, y hacer talleres generales de formación para la enseñanza del inglés $(67 \%$ de los docentes si ha recibido talleres).

Las actividades de entrenamiento en la didáctica general y en inglés, en las que los docentes han participado menos son las siguientes: El $78 \%$ de los profesores, no ha sido capacitado de manera formal en docencia, y ningún profesor ha realizado un postgrado en educación; el $67 \%$ de los profesores no ha realizado algún tipo de 
curso; ningún docente ha realizado el componente docente requerido para impartir clases; el 89\% de los profesores no ha realizado ningún curso de didáctica, ni ha realizado cursos de planificación educativa. El $78 \%$ de los profesores de la institución no ha realizado ninguno curso de evaluación. Además un 56\% no posee formación en el empleo de recursos educativos.

La dimensión Orientación se refiere a todas aquellas sugerencias, recomendaciones e instrucciones recibidas por el docente, directamente de sus superiores y compañeros con mayor experiencia y conocimiento sobre didáctica, y dirigidas a corregir posibles errores y mejorar su desempeño en el aula. En esta dimensión la mediana se ubicó en 37,50 puntos, en la categoría suficiente, lo cual significa que en general el grupo de docentes ha recibido orientaciones dirigidas a potenciar su dominio de la didáctica para la enseñanza del inglés como lengua extranjera. El puntaje mínimo fue 6 puntos sobre 50 y se ubica en la categoría muy deficiente, mientras que el máximo fue 50 en la categoría excelente. La distribución de la dimensión orientación es bastante heterogénea.

En la dimensión orientación, el 77,4\% de los docentes se ubica en las categorías excelente y suficiente, mientras que sólo el $22,22 \%$ tiene una formación entre deficiente y muy deficiente. Esto significa que la gran mayoría de las docentes de la institución investigada han recibido diferentes orientaciones de sus superiores y de otros colegas para utilizar la didáctica. Las áreas en las docentes han recibido más orientación son: cómo mejorar sus destrezas didácticas (67\%); explicaciones sobre el método empleado por la institución (78\%); orientación sobre cómo utilizar el material didáctico (67\%); cómo escoger material apropiado (89\%); acceso a formatos de planificación de la clase (78\%); instrucciones concretas de cómo planificar la clase (78). Por otra parte, las áreas en las que los docentes han recibido menos orientación son: uso de juegos didácticos, $66 \%$; orientación sobre ejercicios (56\%).

La dimensión modelaje y monitoreo se refiere a todas aquellas actividades formativas en las cuales el docente ha participado y ha sido monitoreado por otros profesionales con más experiencia, o también ha tenido la oportunidad de observar a otros colegas expertos en el aula de clase. En esta dimensión la mediana se ubicó en 41,67 puntos, en la categoría excelente, lo cual significa que en general el grupo de docentes ha recibido muy buenos aportes del modelaje $y$ monitoreo por parte de sus superiores y colegas. El puntaje mínimo obtenido fue 8 puntos sobre 50 y se ubica en la categoría muy deficiente, mientras que el máximo fue 50 y está en la categoría excelente. La distribución de la dimensión es heterogénea, ya que el recorrido es muy amplio entre el mínimo y el máximo. En las categorías de Modelaje y monitoreo se observa que el $55,56 \%$ se ubica en las categoría excelente, mientras que $22,22 \%$ hace uso del modelaje $y$ ha sido monitoreado de manera regular. Además un $11,11 \%$ se ubica en la categoría deficiente y otro $11,11 \%$ en la categoría muy deficiente. Esto significa que un poco más de la mitad de los docentes hace uso del modelaje y es monitoreado en clase.

Las actividades de Modelaje y monitoreo en las que los docentes han participado más son aquellas donde los docentes han sido observados por otros profesores o directivos (89\%); aquellas donde se le han hecho observaciones aleatorias de su práctica docente (89\%); a las prácticas supervisadas, un $67 \%$. Las actividades de modelaje y monitoreo en las que los docentes han participado menos 
son: el seguimiento de clases, y la observación clases de inglés de otros profesores.

La dimensión Lectura y revisión de textos se refiere a la revisión de la documentación relacionada con la didáctica integral y a la enseñanza del inglés. En esta dimensión la mediana se ubicó en 16,67 puntos, en la categoría deficiente, lo cual significa que en general el grupo de docentes lee y revisa poco material que les permita estar actualizados en didáctica para la enseñanza del inglés como lengua extranjera. El 22,22\% de los docentes se ubicó en la categoría excelente, mientras que otro $22,22 \%$ se ubicó en la categoría suficiente. Un $33,33 \%$ se ubicó en la categoría deficiente y un $22,22 \%$ se ubicó en la categoría muy deficiente. Esto significa que la mayoría de los docentes de la institución investigada (55,55\%) no tiene hábito de lectura y de revisión de textos relacionados con la enseñanza del inglés. La actividad relacionada con la Lectura y revisión de textos, en la que los docentes han participado con mayor frecuencia es mantenerse actualizados en contacto con material sobre nuevas tendencias de la enseñanza del inglés (67\%). Por otra parte, las actividades relacionadas con la lectura y revisión de textos en las que los docentes han participado menos son: recibir alguna publicación periódica sobre la enseñanza del inglés (67\%).

La dimensión Redes y compartir de experiencias se refiere a todas aquellas actividades formativas en las cuales el docente haya compartido su información sobre la práctica pedagógica con otros docentes y esté incorporado o afiliado a alguna red social como Tesol Internacional o Venezuela Tesol. En esta dimensión la mediana se ubicó en 8,33 puntos sobre 50, en la categoría deficiente, lo cual significa que en general el grupo de docentes participa muy poco en redes para compartir experiencias que le permitan tener más información sobre la didáctica para la enseñanza del inglés como lengua extranjera. El $55,56 \%$ de los profesores se ubica en las categorías muy deficiente y un $11,11 \%$ en la deficiente, mientras que el 22,22\% comparte redes e información de manera regular y solo un $11,11 \%$ se encuentra en la categoría excelente. Esto significa que la gran mayoría de los docentes de la institución investigada no pertenece a redes de información, ni comparte información con otros colegas sobre páginas web o medios para mejorar su práctica educativa.

La actividad vinculada con la pertenencia a redes y el compartir de experiencias en la que los docentes han participado más, es conocer experiencias de expertos internacionales en enseñanza del inglés como lengua extranjera (56\%). Las actividades relacionadas con la pertenencia a redes y el compartir de experiencias en las que los/las docentes han participado menos son las siguientes: un $100 \%$ de los profesores manifestó no ser miembro de ninguna red de profesores o de instituciones relacionadas con la enseñanza del inglés en el país o fuera de él; el $78 \%$ de los profesores objeto de estudio no ha asistido o es miembro de redes o foros de didáctica del inglés; el $67 \%$ no asiste a reuniones con colegas de otras instituciones para compartir experiencias

La dimensión Motivación se refiere a todo lo que le permite a el/la docente enriquecer y aumentar su autoestima y dedicación hacia la labor docente. En esta dimensión la mediana se ubicó en 33,33 puntos, en la categoría suficiente. Todos los docentes, excepto el caso 7 (caso atípico) obtuvieron el mismo puntaje en motivación $(33,33)$, lo cual significa que en general el grupo de docentes está motivado pero no lo suficiente como para participar en otras actividades que pueda ofrecer la institución, 
como se vio en la sinergia anterior, donde sólo el 44\% participaba en reuniones de compartir de experiencias en la institución. El $88,9 \%$ se ubica en la categoría suficiente, mientras que sólo el 11,11\% tiene una categoría deficiente. Esto significa que la gran mayoría de las docentes de la institución investigada están motivadas. Las actividades relacionadas con la motivación en las que los docentes han participado más son recibir reconocimientos e incentivos.

Los resultados obtenidos coinciden con los encontrados en otras investigaciones. $\mathrm{Si}$ bien que el docente egresado en inglés debe estar preparado, para enseñar el idioma partiendo de un enfoque comunicativo (Bracho y Castro, 2007), investigaciones realizadas por expertos en el área, tales como Chacón (2002), Cullen (1998), Sato y Kleinsasser (1999) como se citaron en Salazar y Batista (2005), han encontrado que una gran mayoría de docentes de inglés presentan carencias en cuanto a sus competencias comunicativas y a la metodología para la enseñanza de lenguas. Otros estudios indican que los docentes centran sus prácticas pedagógicas en la enseñanza de aspectos formales de la lengua a partir de ejercicios de memorización y repetición (Chacón, 2002 y Li, 1998 como se citaron en Salazar y Batista, 2005). Lo anterior indica que es necesario que los docentes sean entrenados y capacitados en didáctica, para para emplear adecuadamente de recursos educativos $y$ hacer seguimiento del desempeño de los alumnos y de su progreso, mediante instrumentos de evaluación acordes con lo enseñado.

Existen muchas limitaciones en la formación didáctica de los docentes estudiados, por lo que sería importante avanzar hacia otras investigaciones que permitan explicar los factores que inciden en estos niveles de formación y además desarrollar propuestas formativas en el campo de la didáctica del inglés.

\section{CONCLUSIONES}

En cuanto a la formación acerca de la didáctica de enseñanza del inglés como lengua extranjera, que tienen los docentes del programa bilingüe de primaria se puede decir que el aspecto más deficiente de la formación fue la vinculación a redes y compartir de experiencias, seguida por participación en actividades directas de entrenamiento. Los aspectos de la formación didáctica en los que los docentes tuvieron mayores fortalezas fueron haber sido monitoreados en su trabajo y haber recibido modelaje y orientaciones de otros expertos las cuales se ubicaron en la categoría excelente. Aunque más de la mitad de los docentes tiene pregrado en educación, un porcentaje importante no lo tiene. La mayor formación recibida ha sido en el uso del idioma inglés, aunque un pequeño porcentaje no ha recibido entrenamiento formal en el idioma. Las mayores debilidades están en la formación didáctica para la enseñanza del inglés y en los talleres especiales de planificación, técnicas y evaluación en la enseñanza específica de una lengua extranjera. Otra debilidad encontrada fue la formación recibida a través de la lectura y revisión de la bibliografía relacionada con la didáctica integral y a la enseñanza del inglés, pues en general el grupo de docentes lee y revisa poco material que les permita actualizarse en la didáctica para la enseñanza del inglés como lengua extranjera. Estos resultados sugieren que existe la necesidad de implementar un programa de formación en didáctica para instruir a los docentes es imperioso. 


\section{REFERENCIAS}

Asociación de Supervisición y Desarrollo del Currículo de Estados Unidos (ASCD, 2011) The whole Child. Recuperado http://www.ascd.org/programs/TheWhole-Child/The-Whole-Child.aspx

Banco Interamericano de Desarrollo. (2011) Recuperado de http://www.iadb.org/es/temas/educacion /habilidades-para-el-siglo-xxi-laensenanza-del-ingles-en-costarica,6641.html

Bracho L. y Castro M. C. (2007) El Inglés como Segunda Lengua, Proyecto de Alfabetización de Adultos. (Trabajo de grado). Licenciatura en Educación. Universidad Metropolitana, Caracas

Brewster J, Ellis G, Girard D. (2002) The Primary English Teacher's Guide. England: Pearson Education

Díaz, H. (2009). El inglés y su dominio entre niños y jóvenes. Perú: Consejo Nacional de Educación Recuperado de http://www.cne.gob.pe/index.php/HugoD\%C3\%ADaz/el-ingles-y-su-dominioentre-ninos-y-jovenes.html

Cooper, J. (2006) Estrategias de enseñanza (Guía para una mejor Instrucción). México: Editorial Limusa

Hernán I. y Garcés A. (2006) Didáctica del Inglés para primaria. Colección Didáctica. Madrid: Pearson Prentice Hall
Hernández Sampieri, Fernández Collado y Baptista Lucio (2010). Metodología de la Investigación. México D.F.: Mac Graw-Hill

Hurtado J. (2010) El proyecto de Investigación. Comprensión Holística de la metodología y la investigación. Primera reimpresión. Bogotá-Caracas: Ediciones Quirón. Sypal Servicios y Proyecciones para América Latina

Martínez, F. (2010). Primera Infancia, Bilingüismo y educación Infantil. México: Editorial Trillas

Martínez, A. (2003) Didáctica (Lengua y la literatura) articulo revista., vol.15.139160 Recuperado de http://revistas.ucm.es/edu/11300531/art iculos/DIDA0303110139A.PDF

Medina B, A. (2009). Didáctica de las Lenguas extranjeras (Inglés) Editor Alexander Ortiz Caña. Recuperado de http://www.bubok.com/libros/8016/Dida ctica-de-las-lenguas-extranjeras-Ingles

Medina A. y Mata F. (2002). Didáctica General. Colección Didáctica Pearson Educación, Madrid, España

Salazar. L. y Batista, J. (2005). Hacia la consolidación de un enfoque ecléctico en la enseñanza de idiomas extranjeros. Revista Paradigma. Volumen $26 \mathrm{~N}^{\circ} 1$. Maracay, Venezuela: Universidad Pedagógica Experimental Libertador 